\title{
Congenital cataract-progressive muscular hypotonia-hearing loss-developmental delay syndrome
}

INSERM

\section{Source}

INSERM. (1999). Orphanet: an online rare disease and orphan drug data base. Congenital cataract-progressive muscular hypotonia-hearing loss-developmental delay syndrome. ORPHA:330054

Congenital cataract-progressive muscular hypotonia-hearing loss-developmental delay syndrome is a rare, genetic, mitochondrial myopathy disorder characterized by congenital cataract, progressive muscular hypotonia that particularly affects the lower limbs, reduced deep tendon reflexes, sensorineural hearing loss, global development delay and lactic acidosis. Muscle biopsy reveals reduced complex I, II and IV respiratory chain activity. 